\title{
The Use of CT and MRI in Evaluation of Primary Brain Tumors in Saudi Arabia
}

\author{
Saad Mohammed Alhaqbani ${ }^{*}$, Dalal Aayed Al-Harbi ${ }^{2}$, Abdulaziz Musaad Mutiran Aljohani ${ }^{3}$, \\ Talal Ahmad Ali Alomari ${ }^{4}$, Mohammed Mousa Alghamdi ${ }^{5}$, Fawaz Abdulrahman Alsaleh ${ }^{6}$, \\ Eman Mohammad Alfaraj ${ }^{7}$, Abdulrahman Nasser Alakeel ${ }^{8}$
}

${ }^{1}$ Prince Sattam Bin Abdulaziz University, Al-Kharj, ${ }^{2}$ Batterjee Medical College, Jeddah, ${ }^{3}$ King Khalid University, Abha, ${ }^{4} \mathrm{Al}-\mathrm{Baha}$ University, Saudi Arabia ${ }^{5}$ Almaarefa Colleges, Riyadh, ${ }^{6} \mathrm{Al}$ Imam Mohammed Ibn Saud Islamic University, Riyadh, ${ }^{7}$ ImamAbdulrahman Bin Faisal University, Dammam, ${ }^{8}$ King Saud Bin Abdulaziz University for Health Sciences, Riyadh, Saudi Arabia

*Corresponding Author: Saad Mohammed Alhaqbani, E-mail: S3dm92@hotmail.com

\begin{abstract}
Background: Imaging plays a crucial function in the management of patients with brain tumors. The technical improvement of computed tomography (CT) and magnetic resonance imaging (MRI) with the advancement of, brand-new imaging techniques highly enhanced the detection and characterization of brain tumors. This comprehensive review of literature is aimed to discuss the roles of MRI \& CT in the diagnosis and evaluation of primary brain tumors, we attempted to discuss the advantage and disadvantage of each modality and which is more effective in this matter.

Objective: to find a relevant article to our study, this is discussing the roles of MRI and CT in evaluation of Primary brain tumors. Several terms were used in the search through the databases; (MRI, CT, and Imaging), combined with (Brain cancer, primary brain tumors, glioblastomas).

Method: We performed a comprehensive search of literatures among main medical databases; PubMed (MIDLINE), Embase, and science direct, up to November 2017, We limited our search to English language studies, and only to Human trails.

Results: Computed tomography (CT) might be the very first modality employed in a patient presenting with a brain tumor but for one of the most part MRI is the primary imaging modality in brain tumor patients. The function of CT is mostly relegated to emerging imaging in the detection of hemorrhage, herniation, and hydrocephalus but mass effect from brain tumors and calcification within brain tumors such as oligodendrogliomas or meningiomas can potentially be discovered.

Conclusion: The main use of MRI-based medical image analysis for brain tumor studies remains in medical diagnosis, patient tracking and treatment preparation, however it could also be useful in clinical trials.
\end{abstract}

\section{INTRODUCTION}

Imaging plays a crucial function in the management of patients with brain tumors. The technical improvement of computed tomography (CT) and magnetic resonance (MRI) with the advancement of, brand-new imaging techniques highly enhanced the detection and characterization of brain tumors. For the ideal therapeutic management of the oncologic patient not only the recognition of the lesion is needed, however likewise the exemption of other diseases that can mimic brain tumors ${ }^{(1,2)}$. Imaging plays an essential role in the assessment of patients with brain tumors. CT and MRI represent the two crucial and frequently used imaging modalities. They have a significant impact on patient care. The technical enhancement of CT and MRI, the energy of contrast material in the imaging of brain tumors along with the introduction of new imaging strategies, enhanced substantially the detection and the evaluation of brain neoplasms. As soon as a brain tumor is scientifically presumed, radiologic examination is required to figure out the location, the level of the tumor and its relationship to the surrounding structures ${ }^{(3,4)}$. Brain tumors share some functions and obstacles for medical diagnosis and treatment with tumors elsewhere in the body, but they also posture specific problems that relate to the distinct homes of the organ they being in ${ }^{(5)}$. Most of the brain is separated from the blood 
by the blood-- brain barrier (BBB) that applies a lot more restrictive control over compounds that are enabled to pass (or may even undergo help with transportation) than most other organs. Thus, lots of tracers that quickly reach tumors in the body would reach brain tumors only once the tumor caused a disturbance of the $\mathrm{BBB}$, for example glioblastomas; when the tumor established from intracranial tissues that do not have a BBB, for example meningioma (derived from the meninges); or for brain metastasis (seeding from within the capillary) (5). The World Health Organization (WHO) defines four grades of brain tumors based mainly upon tumor aggressiveness with grade I tumors being relatively non-aggressive and grade IV tumors being extremely aggressive (6). Traditionally with the first edition in 1979 , WHO grades were appointed on the basis of histologic functions such as mitotic activity, necrosis, and infiltration ${ }^{(7)}$. The second edition, in 1993, included immunohistochemistry, and the third edition in 2000 bundled genetic profiles into the meanings of brain tumors ${ }^{(8,9)}$. The most recent WHO central nerve system (CNS) tumor classification system was released in 2007 and continues to integrate genetic profiles into tumor definitions and histological variations (6). Genetics and molecular profiles of brain tumors continue to be an active area of research study with diagnostic, prognostic, restorative, and imaging ramifications ${ }^{(10,11)}$.

This comprehensive review of literature is aimed to discuss the roles of MRI \& CT in the diagnosis and evaluation of primary brain tumors, we attempted to discuss the advantage and disadvantage of each modality and which is more effective in this matter.

\section{METHODOLOGY}

We performed a comprehensive search of literatures among main medical databases; PubMed (MIDLINE), Embase, and science Direct, up to November 2017, to find a relevant article to our study which is discussing the roles of MRI and CT in evaluation of Primary brain tumors. Several terms were used in the search through the databases; (MRI, CT, and Imaging), combined with (Brain cancer, primary brain tumors, glioblastomas). We limited our search to
English language studies, and only to Human trails

\section{RESULTS \& DISCUSSION}

With a prevalence of less than $1 \%$ in the western population, brain tumors are not typical; nevertheless, they are among the most fatal cancers ${ }^{(12)}$. A recent research study estimated the US occurrence rate for main tumors of the brain or nervous system to be around 25 per 100000 grownups with approximately one-third of the tumors being malignant and the rest either borderlinemalignant or benign ${ }^{(13)}$.

The standard technique for the brain tumor diagnosis is MRI ${ }^{(12,14)}$. MRI is a non-invasive method, which offers good soft tissue contrast ${ }^{(15)}$ and is widely readily available in centers. It is utilized in combination with other imaging methods, such as computed tomography (CT), positron emission tomography (PET) and magnetic resonance spectroscopy (MRS) to supply the most precise info about tumor morphology and metabolic process. MRI remains the accepted standard and for that reason we will focus on MRI-based methods $(14,15)$

\section{Computed Tomography:}

Computed tomography (CT) was introduced in the clinical practice in 1972 and rapidly became an essential consider the radiological diagnosis. With the arrival of CT in neuroradiology direct images of the brain could be produced, and a new era in cerebral research studies started. CT of the brain, which became the treatment of choice for examination and diagnosis of brain tumors, has emplaced intrusive procedures such as pneumoencephalography or cerebral angiography ${ }^{(14,16)}$. Progressive enhancement of the image quality, decrease of costs, and reduction of scan times has actually resulted in significant expansion of CT applications. The utility of contrast product in the imaging of the brain improved the efficacy of $\mathrm{CT}$ in the diagnosis of brain tumors. Enhancement is the increased difference in an imaging particular between a sore and surrounding typical tissue after administration of contrast agent. This is due to the disruption of the blood-brain barrier (BBB) of the tumor vessels, which allows the passage of the contrast material into the extracellular spaces of the tumor (Fig. 1) ${ }^{(18)}$. 


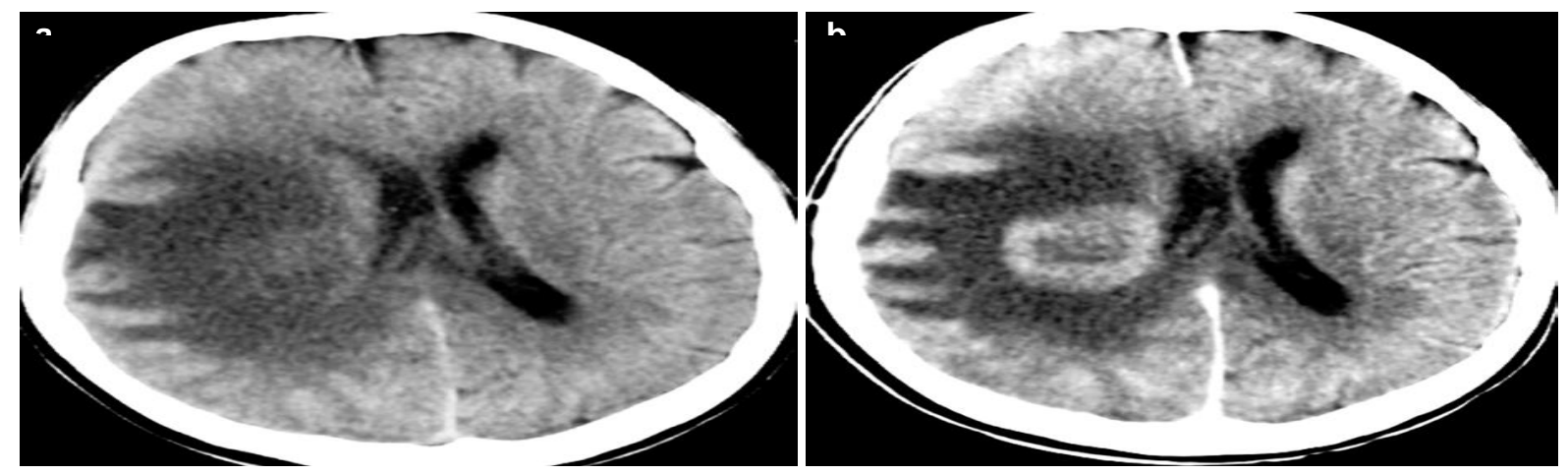

Fig. 1: Enhancement of tumor after IV contrast administration, contrast material identifies areas of BBB disruption facilitating thedisruption. (Left) CT before and (Right) after contrast administration. The detection of the neoplastic tissue neoplasm is clearly demonstrated on post-contrast $\mathrm{CT}^{(16)}$.

On CT, the increased concentration of the contrast material within the tumor interstitium leads to higher attenuation values within the tumor than in the surrounding brain. Most of the brain tumors improve after the administration of contrast product. The enhancement attributes of different types of brain tumors will be gone over in the following chapters. Progress in CT development continued rapidly and new innovation has revolutionized the field. Spiral and multislice CT allow faster acquisition times with substantially improved 3D spatial resolution. CT angiography provides images of excellent quality in a noninvasive way and is of great importance in the assessment of the relationship between the tumor and the vessels. Perfusion imaging techniques enable

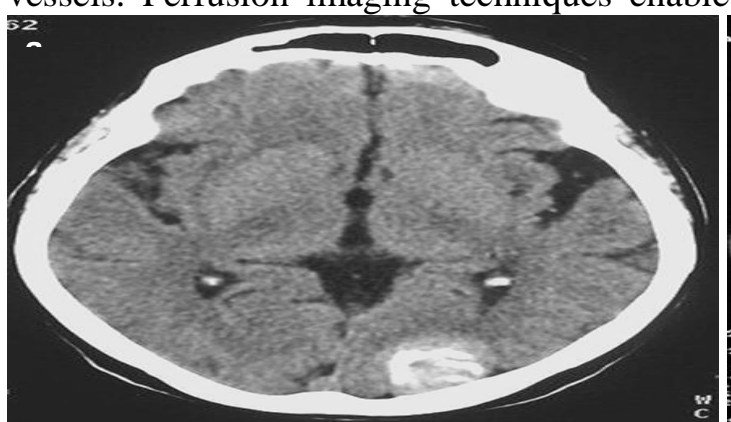

Fig. 2: CT versus MRI in calcified meningioma. (a) Axial CT. (b) Axial T1-weighted MRI. Densely calcified tumor is clearly demonstrated on CT. Most calcification is isointense to brain on T1WI.

\section{Magnetic Resonance Imaging:}

Magnetic resonance imaging (MRI) is the method of option for assessing patients who have signs and signs recommending a brain tumor. Its multiplanar capability exceptional contrast resolution and versatile procedures enable it to play an essential function in assessing tumor area and extent, in directing biopsies, in planning the appropriate treatment,

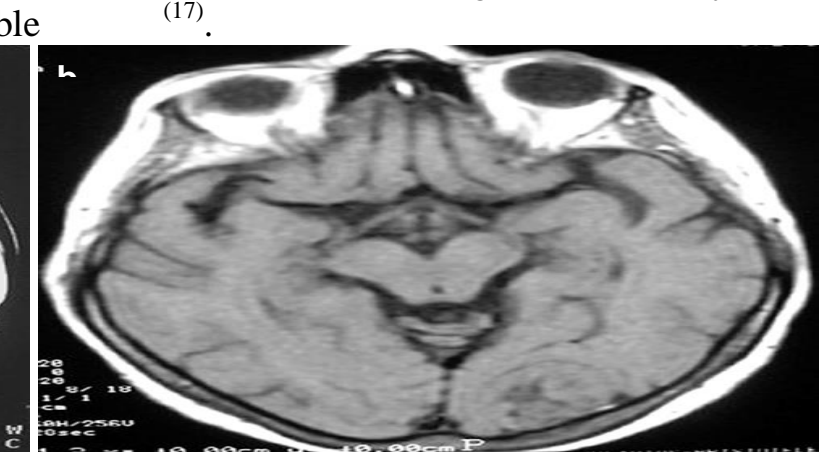

accurate measurement of $\mathrm{CBV}$ and $\mathrm{CBF}$ values in a variety of clinical and experimental settings ${ }^{(16)}$.

CT-guided stereotactic biopsy is a reliable method for histological diagnosis of brain tumors and showed to be valuable in planning the appropriate treatment for each patient.

Although MR is the main diagnostic tool for dis- eases of the central nervous system, CT is still a valuable modality in the imaging of brain tumors. CT is superior in detecting calcification, hemorrhage, and in evaluating bone changes related to a tumor (Fig.2). Patients with pacemakers or metallic devices as well as critically ill, pediatric or unstable patients represent some of the specific areas where CT is the diagnostic modality of choice (17) and in evaluating the therapeutic results. The standard strategy for the brain tumor medical diagnosis is MRI ${ }^{(12,14)}$. MRI is a non-invasive method, which offers great soft tissue contrast ${ }^{(15)}$ and is commonly available in clinics. MRI makes it possible to produce considerably different kinds of tissue contrast by differing excitation and repeating times, makings it a really versatile tool for imaging various 
structures of interest. Due to the nature and look of brain. Tumors, one MRI series is not adequate to fully sector the tumor including all its sub-regions. In present medical regimen, different MRI series are used for the diagnosis and delineation of tumor compartments ${ }^{(19)}$. These sequences consist of T1-weighted MRI (T1), T1-weighted MRI with contrast enhancement (T1c), T2-weighted MRI (T2) and T2-weighted MRI with fluid-attenuated inversion healing (T2FLAIR); however, acquisition specifications of these modalities are not standardized. Patients with gliomas are normally taken a look at by the previously described MR imaging procedures according to the action assessment in neuro-oncology (RANO) standards with a slice thickness of $\leq$ $5 \mathrm{~mm}$ without a space in between the pieces (20). For volumetry, high-resolution 3D volume images are carried out, consisting of a minimum of contrast-enhanced T1-weighted images with isotropic resolution. (Fig. 3) shows an axial slice of the 4 standard series for a glioma patient consisting of manually drawn tumor areas.
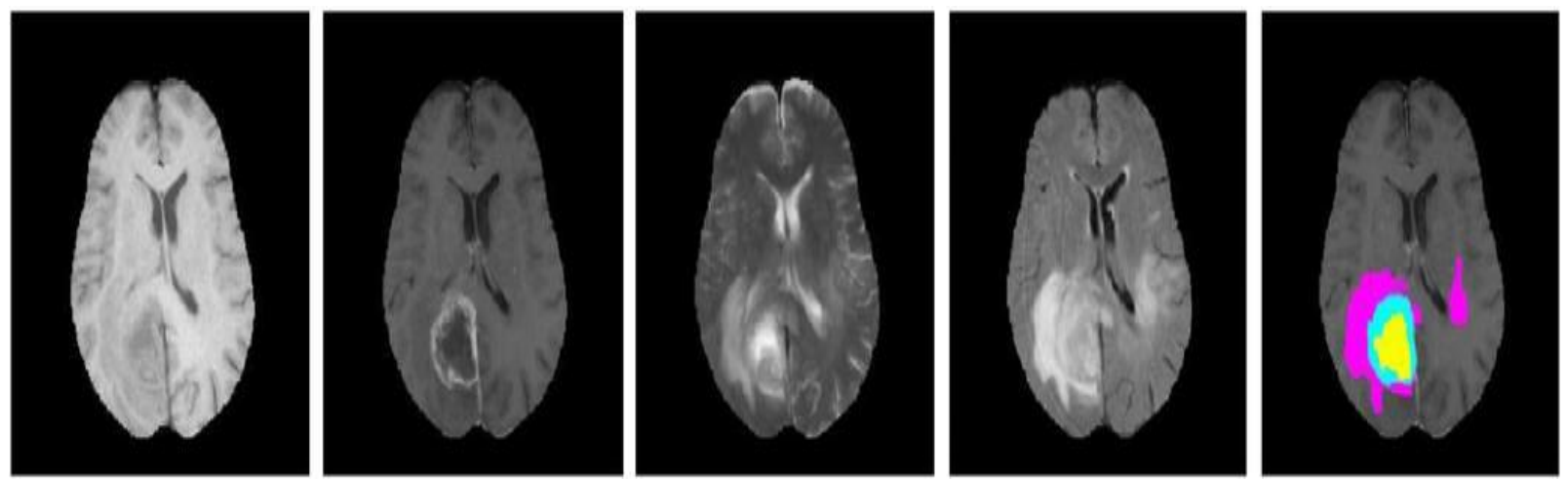

Fig. 3: One axial slice of an MR image of a high-grade glioma patient. From left to right: T1weighted image, T1-weighted image with contrast enhancement, T2-weighted image, T2FLAIRweighted image and manual segmentation into necrotic (yellow), active (green), edema (pink) tumor compartments. Necrosis and active tumor regions were segmented based on the T1-weighted image with contrast enhancement, whereas the edema region was segmented based on the registered T2FLAIR-weighted image ${ }^{(15)}$.

\section{CONCLUSION}

Computed tomography (CT) might be the very first modality employed in a patient presenting with a brain tumor but for one of the most part MRI is the primary imaging modality in brain tumor patients. The function of CT is mostly relegated to emerging imaging in the detection of hemorrhage, herniation, and hydrocephalus but mass effect from brain tumors and calcification within braintumors such as oligodendrogliomas or menigiomas can potentially be discovered. The main use of MRI-based medical image analysis for brain tumor studies remains in medical diagnosis, patient tracking and treatment preparation, however it could also be useful in clinical trials. The division is vital for keeping track of tumor growth or shrinking in patients throughout treatment, for tumor volume measurements and it also plays an essential function in surgical planning or radiotherapy planning, where not only the tumor has to be detailed, but also surrounding healthy structures are of interest.

\section{REFERENCES}

1. Bruzzone MG, D'Incerti L, Farina LL, Cuccarini V, Finocchiaro G (2012): CT and MRI of brain tumors. Q J Nucl Med Mol Imaging, 56(2):112-37.

2. Cha $\mathbf{S}$ (2006): Update on brain tumor imaging: from anatomy to physiology. AJNR Am J Neuroradiol.,27: 475-487.

3. Belden CJ, Valdes PA, Ran C et al. (2011): Genetics of glioblastoma: a window into its imaging and histopathologic variability. Radiographics, 31:1717-1740.

4. Bangiyev L, Rossi Espagnet MC, Young $R$ et al. (2014): Adult brain tumor imaging: state of the art. SeminRoentgenol., 49:39-52.

\section{Herholz K, Langen K-J, Schiepers}


C, Mountz JM (2012): Brain Tumors. Seminars in nuclear medicine,42(6): 356-370.

6. Louis DN, Ohgaki H, Wiestler OD et al. (2007): The 2007 WHO classification of tumours of the central nervous system.ActaNeuropathol., 114:97-109.

7. Zulch KJ (1979): Histological typing of tumours of the central nervous system. Geneva: World Health Organization.

https://www.springer.com/gp/book/97 83540569718

8. Kleihues P, Sobin LH (2000): World Health Organization classification of tumors. Cancer, 88:2887.

9. Kleihues P, Burger PC, Scheithauer BW (1993): The new WHO classification of brain tumours. Brain Pathol., 3:255-268.

10. Gupta K, Salunke $P$ (2012): Molecular markers of glioma: an update on recent progress and perspectives. J Cancer Res ClinOncol., 138:1971-1981.

11. Cancer Genome Atlas Research Network (2008): Comprehensive genomic characterization defines human glioblastomagenes and core pathways. Nature, 455:1061-1068.

12. DeAngelis LM (2001): Brain tumors N. Engl. J. Med., 344: 114-23.

13. Kohler B A, Ward E, McCarthy B J, Schymura M J, Ries L A G., Eheman C, Jemal A, Anderson R N, Ajani U A and Edwards B K (2011): Annual report to the nation on the status of cancer, 1975-2007, featuring tumors of the brain and other nervous system J. Natl Cancer Inst., 103 71436.
14. Wen $P$ Y et al. (2010): Updated response assessment criteria for highgrade gliomas: response assessment in neuro-oncology working group J. Clin. Oncol., 28: 1963-72.

15. Liang ZP, Lauterbur PC (2000): Principles of Magnetic Resonance Imaging: A Signal Processing Perspective ,New York: Wiley.

16. Mabray MC, Barajas RF, Cha $S$ (2015): Modern Brain Tumor Imaging. Brain Tumor Research and Treatment, 3(1): 8-23.

17. Nabavi DG, Cenic A, Craen RA et al. (1999): CT assessment of cerebral perfusion: experimental validation and initial clinical experience. Radiology, 213:141-149.

18. Whelan HT, Clanton JA, Wilson RE et al. (1988): Comparison of CT and MRI brain tumor imaging using a canine glioma model. Pediatr Neurol.,4(5):279-283.

19. Drevelegas A and Papanikolaou $\mathbf{N}$ (2011): Imaging modalities in brain tumors Imaging of Brain Tumors with Histological Correlations ed A Drevelegas ,Berlin: Springer, chapter 2 ,pp 13-34.

20. Pope W B and Hessel C (2011): Response assessment in neurooncology criteria: implementation challenges in multicenter neurooncology trials Am. J. Neuroradiol., 32 794-7. 